\title{
Análise da produção científica da Revista Brasileira de Geriatria e Gerontologia: uma revisão bibliométrica
}

\author{
Analysis of the scientific production of the Brazilian Journal of Geriatrics and Gerontology: a \\ bibliometric review
}

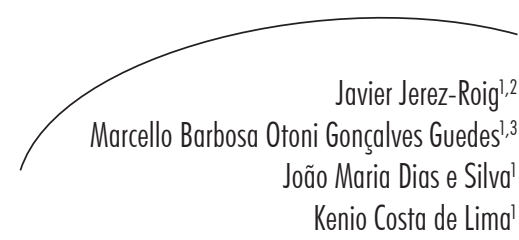

\section{Resumo}

Objetivo: Descrever e analisar o perfil das publicações da Revista Brasileira de Geriatria e Gerontologia (RBGG) no período de 2006 a 2013. Método: Trata-se de pesquisa de revisão bibliográfica, do tipo descritivo, com análise bibliométrica da produção científica da RBGG. A coleta dos dados foi realizada por três pesquisadores treinados. Contatou-se a equipe editorial via correio eletrônico para obter a informação referente ao número de manuscritos recebidos, rejeitados e aceitos na revista. Resultados: Publicou-se um total de 400 trabalhos, dos quais $301(75,3 \%)$ foram artigos originais e $42(10,5 \%)$ revisões. A maior parte dos trabalhos $(95,1 \%)$ é de autores brasileiros, predominando as Regiões Sudeste $(43,4 \%)$ e Sul $(26,8 \%)$, especificamente nos estados de São Paulo $(19,8 \%)$, Rio Grande do Sul $(15,6 \%)$ e Rio de Janeiro (12,0\%). A maioria dos trabalhos é de abordagem quantitativa e o tipo de estudo mais frequente é o transversal $(45,9 \%)$, seguido pela revisão de literatura $(13,7 \%)$ e a série de casos de tipo observacional $(10,7 \%)$. Verificou-se, ainda, aumento do tempo para publicação entre 2006 e 2011, seguido por uma estabilidade até 2013. As temáticas mais comuns foram as referentes aos aspectos psicológicos do envelhecimento, atividade física e nutrição. Conclusões: Observou-se aumento continuado no número de trabalhos recebidos e publicados na RBGG durante todo o período. A produção é focada nas instituições universitárias, em âmbito nacional, e centralizada nas regiões Sudeste e Sul. Identificou-se ainda uma grande abrangência de temáticas.

\section{Abstract}

Objective: To describe and analyze the profile of publications of the Brazilian Journal of Geriatrics and Gerontology (RBGG) between years 2006 to 2013. Method: This is a survey of literature review, descriptive type with bibliometric analysis of scientific production of RBGG. Data collection was performed by three trained investigators. The editorial

Palavras-chave: Publicações Periódicas como Assunto. Publicações Científicas e Técnicas. Bibliometria; Envelhecimento.

\footnotetext{
Programa de Pós-graduação em Saúde Coletiva, Departamento de Odontologia. Universidade Federal do Rio Grande do Norte. Natal, RN, Brasil.

2 Serviço de Reabilitação. Hospital Can Misses. Ibiza, Espanha.

3 Curso de Fisioterapia, Faculdade de Ciências da Saúde do Trairi. Universidade Federal do Rio Grande do Norte. Santa Cruz, RN, Brasil.

Correspondência / Correspondence

Javier Jerez-Roig

E-mail: javijerez81@hotmail.com
} 
staff was contacted via e-mail to get information regarding the number of manuscripts received, rejected and accepted. Results: It published a total of 400 articles, of which 301 (75.3\%) were original articles and $42(10.5 \%)$ reviews. Most studies (95.1\%) were published by Brazilian authors, most of whom are from the Southeast (43.4\%) and South (26.8\%), concentrated in the states of São Paulo (19.8\%), Rio Grande do Sul $(15.6 \%)$ and Rio de Janeiro (12.0\%). Most works had quantitative approach and the most frequent type of study is the cross-sectional (45.9\%), followed by literature review $(13.7 \%)$ and a number of observational cases $(10.7 \%)$. There was an increase in time of publication between 2006 and 2011, followed by stability until 2013. The most common themes were related to the psychological aspects of aging, physical activity and nutrition. Conclusions: There has been observed continuing increase in the number of papers received and published in RBGG in this period. The production is focused mainly in the universities at the national scope in the Southeastern and Southern regions. A wide range of topics was also identified.
Key words: Periodicals as Topic. Scientific and Technical Publications. Bibliometrics. Aging.

\section{INTRODUÇÃO}

O processo de envelhecimento vem sendo amplamente discutido por especialistas de diferentes áreas e disciplinas. ${ }^{1}$ Espaços para a publicação e divulgação da produção científica na área do envelhecimento são de extrema importância para a consolidação do conhecimento. A missão de uma publicação científica é contribuir para a construção do edifício teórico do seu campo. ${ }^{2}$

Nessa perspectiva, em 1998, a Universidade Aberta da Terceira Idade da Universidade do Estado do Rio de Janeiro (UnATI-UERJ) deu um passo importante para a consolidação de seu projeto editorial, oferecendo ao leitor a série Textos sobre Envelhecimento. Essa publicação buscou atender à demanda crescente por parte de pesquisadores, pós-graduandos e demais estudiosos interessados em obter subsídios para a realização de suas pesquisas dentro dessa desafiadora área de interesse: o processo de envelhecimento. ${ }^{3}$

Como consequência do crescimento da produção cientifica na área e com o objetivo de difundir o conhecimento e atender à demanda por uma revista científica indexada e de padrão internacional, em 2006 se iniciou a Revista Brasileira de Geriatria e Gerontologia (RBGG), uma continuação do título Textos sobre Envelhecimento. Sua concepção levou em consideração o escopo e a complexidade do envelhecimento humano, reclamando uma superação dos modelos predominantes no país. ${ }^{4}$

Em oito anos de existência, a RBGG se consolidou como uma das mais importantes revistas científicas na área do envelhecimento no Brasil e conseguiu a indexação em bases de dados como Literatura Latino-Americana e do Caribe em Ciências da Saúde (LILACS), Sistema Regional de Informação em Linha para Revistas Científicas da América Latina, Caribe, Espanha e Portugal (Latindex) e Directory of Open Access Journals (DOAJ). ${ }^{5}$ Neste sentido, o maior logro da revista foi a entrada, no ano 2012, no seleto grupo de periódicos brasileiros que fazem parte da Coleção SciELO Brasil (Scientific Eletronic Library Online), ampliando seu alcance de modo geométrico. ${ }^{6}$

Atualmente, a RBGG é qualificada, segundo a Coordenação de Aperfeiçoamento de Pessoal de Nível Superior (CAPES), no grupo B1 na área Interdisciplinar; B2 em Educação Física e Saúde Coletiva; B3 em Enfermagem, Psicologia e Sociologia; B4 nas áreas da Medicina I, II e III, Serviço Social e Ciências Ambientais; e B5 nas áreas da Farmácia e Engenharias I, confirmando sua importância como um dos principais veículos de divulgação do conhecimento científico no campo do envelhecimento na visão de temáticas variadas.? 
Além dos índices de qualificação, indicadores que ajudem a traduzir a produção científica na área do envelhecimento tornam-se extremamente importantes e, nesse contexto, a bibliometria deve ser utilizada como metodologia para a obtenção de indicadores de avaliação da produção científica. ${ }^{8}$ De forma geral, o princípio da bibliometria é analisar a produtividade científica ou técnica pelo estudo quantitativo das publicações de determinada temática ou veículo, e seu principal objetivo é o desenvolvimento de indicadores que possam traduzir um panorama da atualidade ou indicar uma tendência, importante para avaliação do processo e grau de desenvolvimento científico e tecnológico. ${ }^{8-10}$

Os artigos publicados em periódicos científicos apresentam dimensões que podem ser medidas por indicadores, dos quais se esperam obter informações relevantes, tais como: volume de investimentos, a proporção de profissionais de nível superior no mercado de trabalho, a quantidade de mestres e doutores titulados, coeficientes técnicos de pesquisadores..$^{11}$ Ainda, pode-se afirmar que os estudos bibliométricos têm sido cada vez mais requisitados para identificargrupos e áreas de excelência acadêmica, destacando-se a importância crescente do papel de demandas e atores extra-acadêmicos na dinâmica da formação de recursos humanos para o mercado e, em particular, sobre o papel dos periódicos científicos neste processo de formação. ${ }^{11,12}$

Sendo assim, o objetivo do presente estudo foi descrever e analisar o perfil das publicações da Revista Brasileira de Geriatria e Gerontologia (RBGG) no período de 2006 a 2013.

\section{METODOLOGIA}

Trata-se de uma pesquisa de revisão bibliográfica, de tipo descritivo, com análise bibliométrica da produção científica da RBGG entre janeiro de 2006 e dezembro de 2013. Os artigos completos publicados entre os anos 2006 e 2011 foram disponibilizados no sítio da
UnATI, e os produzidos entre 2012 e 2013, na web da SciELO.

Adotaram-se como critérios de inclusão os artigos originais, de revisão, estudos de caso, ensaios, artigos de opinião, de atualização, de reflexão e de comunicação breve, publicados na revista durante o período descrito. Excluíramse, para a análise bibliométrica, os anais de congressos, resenhas, editoriais, cartas ao editor, dissertações e teses. Foi revisado um total de 400 publicações distribuídas em 27 números. Cabe ressaltar que os tipos de publicação editorial, carta ao editor, ensaio, comunicação breve, artigo de opinião, de reflexão e de atualização apenas foram considerados na análise descritiva da variável "tipo de publicação".

A coleta dos dados foi realizada por três pesquisadores, previamente treinados, para assegurar a uniformidade de critérios. Em caso de dúvida, os casos foram consultados coletivamente e discutidos com um quarto pesquisador, coordenador do trabalho. Ao final da escrita, dois bibliotecários da biblioteca do Programa de Pós-Graduação em Saúde Coletiva da Universidade Federal do Rio Grande do Norte (UFRN) revisaram o manuscrito e forneceram um parecer técnico sobre as informações contidas para adequações mais precisas do artigo.

Nesta pesquisa foram considerados quatro grupos de variáveis: referentes à identificação da publicação, aos autores, ao tipo de estudo e variáveis bibliométricas. Primeiramente, mediante leitura do resumo, coletaram-se informações básicas sobre os trabalhos, como número, ano e tipo de publicação, incluindo as seguintes categorias: artigo original, de revisão, estudo/relato de caso, editorial, artigo de opinião, ensaio, artigo de atualização, de reflexão, comunicação breve e outros.

As variáveis referentes à autoria foram: número de instituições envolvidas, número de autores por artigo, nacionalidade destes (brasileira, estrangeira ou mista) e financiamento (sim ou não). Além disso, foram coletadas informações sobre $\mathrm{O}$ 
primeiro autor: formação, instituição, região e estado. Para conhecer a formação acadêmica, foram pesquisados os currículos dos autores na Plataforma Lattes, e em caso de possuir mais de um curso de graduação, considerou-se aquele mais vinculado à temática do artigo.

Informações específicas sobre o trabalho foram obtidas mediante leitura do resumo e texto completo. As informações coletadas foram abordagem (quantitativa, qualitativa $e$ mista), desenho de estudo, temática do trabalho, número da amostra total de cada estudo (para os estudos de revisão, a amostra foi considerada como o número de artigos analisados no estudo), presença ou ausência de análise inferencial, número total de referências bibliográficas e número de referências anteriores aos cinco anos a partir do ano de publicação do artigo.

Os tipos de estudo segundo o desenho foram: estudo de caso, série de casos observacionais (com amostra entre dois e 29 indivíduos), série de casos intervencionais (amostra entre dois e 29), transversal (amostra de 30 ou mais indivíduos), ensaio clínico, estudo ecológico, estudo de casocontrole, estudo de coorte, de revisão e estudo de tradução/validação de questionários. Para o levantamento da temática dos artigos, foi inicialmente criada uma classificação abrangente, por consenso entre os pesquisadores, que se revisou durante o processo de coleta de dados e que resultou em 27 temas ou conjuntos de temas. Categorias temáticas com frequência absoluta menor que cinco foram recategorizadas como "outras temáticas", ou então reclassificadas com outra categoria semelhante, quando possível. Foram consideradas as seguintes categorias: aspectos psicológicos do envelhecimento (incluindo percepções e aposentadoria), atividade física, funcionalidade e fragilidade, atenção primária, fisioterapia (e reabilitação), aspectos farmacológicos, epidemiologia e saúde pública, doenças infecciosas, reumatologia, qualidade de vida (bem-estar), nutrição (inclui antropometria e deglutição), cognição (capacidade cognitiva), quedas e equilíbrio (inclui coordenação e alterações sensoriais), incontinência urinária, institucionalização, fisiologia, cuidados e suporte social, saúde bucal e outras temáticas.

Para conhecer o grau de difusão científica das publicações da $\mathrm{RBGG}$, criou-se um ranking com base no número de citações dos trabalhos. Estas informações se encontram disponíveis no Google Acadêmico.

Por último, coletaram-se ainda informações referidas ao próprio processo editorial, como o tempo (em meses) transcorrido entre o recebimento do manuscrito e sua aprovação e publicação. Ainda considerou-se o número anual de manuscritos recebidos, recusados e aceitos, mediante contato, via correio eletrônico, com o corpo editorial da revista.

Inicialmente, utilizaram-se planilhas do programa Microsoft Excel $\AA$ para a coleta dos dados, de maneira codificada. Posteriormente, os dados foram organizados e analisados mediante o Stata versão 10.0, e mostrados por meio de análise descritiva, com apoio de tabelas e gráficos.

\section{RESULTADOS}

No período 2006 a 2013, foi publicado na RBGG um total de 400 artigos científicos: 301 artigos originais $(75,3 \%), 42$ revisões $(10,5 \%), 28$ editoriais $(7,0 \%)$, oito estudos de caso $(2,0 \%)$, sete artigos de reflexão $(1,8 \%)$, seis cartas ao editor $(1,5 \%)$, cinco artigos de atualização $(1,3 \%)$, um artigo de opinião $(0,3 \%)$, um ensaio $(0,3 \%)$ e uma comunicação breve $(0,3 \%)$. Após aplicar os critérios de exclusão, foram identificados 366 artigos: 107 artigos no período 2006-2009 e 259 artigos no período 2010-2013.

$\mathrm{Na}$ tabela 1, observam-se os resultados das variáveis referentes à autoria dos artigos: financiamento, número de instituições envolvidas, número e nacionalidade dos autores, assim como afiliação, região, estado e formação do primeiro autor. Na variável "afiliação", constam as instituições com oito ou mais publicações na revista e em "formação", aquelas profissões da saúde com frequência maior que 10. 
Tabela 1. Análise descritiva das variáveis referentes à autoria dos artigos publicados na RBGG entre 2006 e 2013. Natal-RN, 2014.

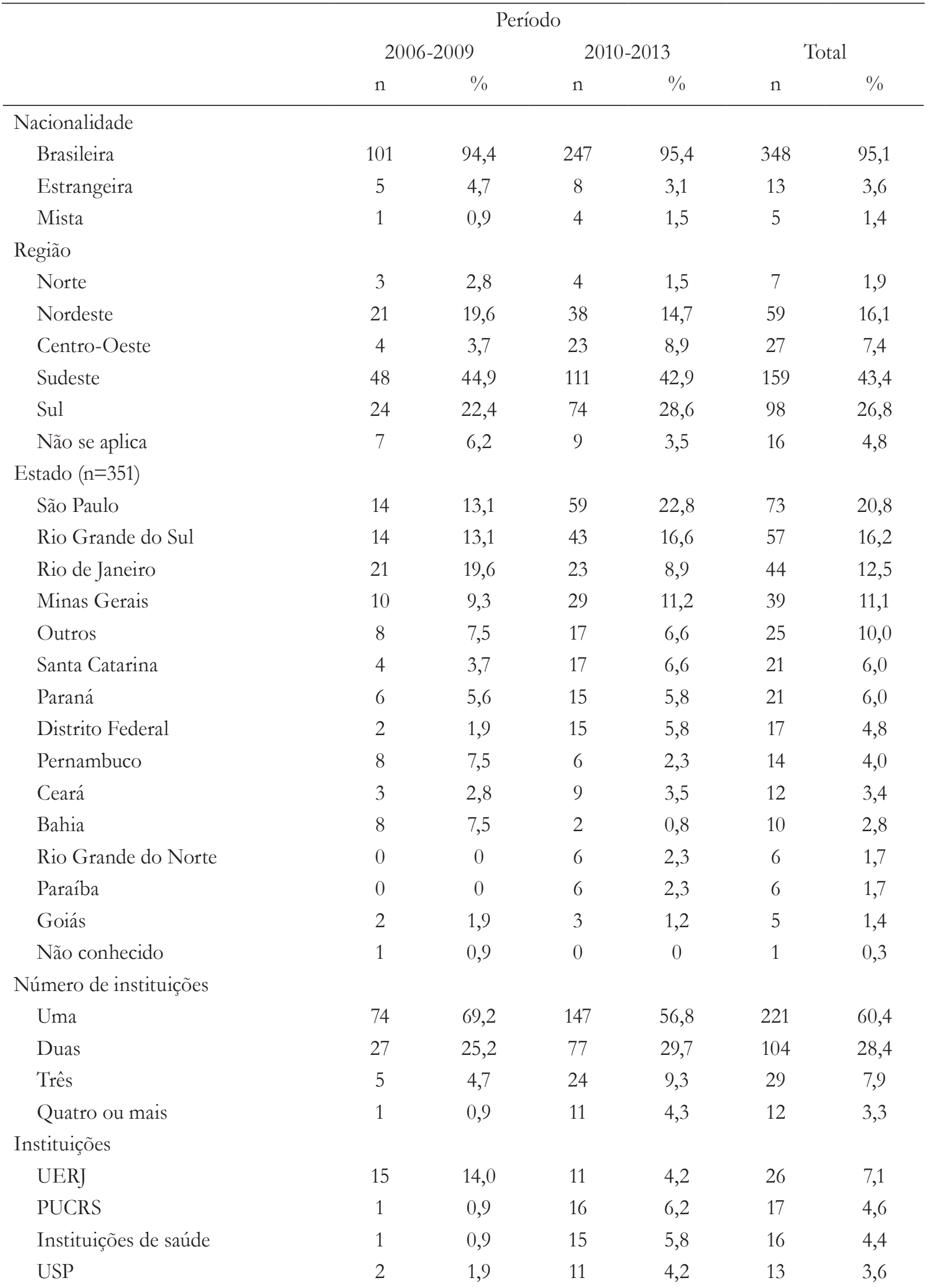




\begin{tabular}{|c|c|c|c|c|c|c|}
\hline & \multicolumn{4}{|c|}{ Período } & & \\
\hline & \multicolumn{2}{|c|}{ 2006-2009 } & \multicolumn{2}{|c|}{$2010-2013$} & \multicolumn{2}{|c|}{ Total } \\
\hline & $\mathrm{n}$ & $\%$ & $\mathrm{n}$ & $\%$ & $\mathrm{n}$ & $\%$ \\
\hline $\mathrm{UCB}$ & 2 & 1,9 & 10 & 3,9 & 12 & 3,3 \\
\hline UFMG & 6 & 5,6 & 5 & 1,9 & 11 & 3,0 \\
\hline UNESP & 2 & 1,9 & 9 & 3,5 & 11 & 3,0 \\
\hline UNIFESP & 4 & 3,7 & 5 & 1,9 & 9 & 2,5 \\
\hline UNICAMP & 0 & 0 & 9 & 3,5 & 9 & 2,5 \\
\hline UESB & 6 & 5,6 & 2 & 0,8 & 8 & 2,2 \\
\hline Outras brasileiras & 62 & 57,9 & 157 & 60,6 & 219 & 59,8 \\
\hline Outras estrangeiras & 6 & 5,6 & 9 & 3,5 & 15 & 4,1 \\
\hline \multicolumn{7}{|l|}{ Financiamento } \\
\hline $\operatorname{Sim}$ & 11 & 10,3 & 34 & 13,1 & 45 & 12,3 \\
\hline Não & 96 & 89,7 & 225 & 86,9 & 321 & 87,7 \\
\hline \multicolumn{7}{|l|}{ Número de autores } \\
\hline $1-2$ & 36 & 33,6 & 62 & 23,9 & 98 & 26,8 \\
\hline $3-4$ & 42 & 39,2 & 106 & 40,9 & 148 & 40,4 \\
\hline 5 e mais & 29 & 27,1 & 91 & 35,1 & 120 & 32,8 \\
\hline \multicolumn{7}{|c|}{ Formação do primeiro autor } \\
\hline Fisioterapia & 20 & 18,7 & 53 & 20,5 & 73 & 19,9 \\
\hline Educação Física & 13 & 12,1 & 39 & 15,1 & 52 & 14,2 \\
\hline Enfermagem & 16 & 15,0 & 26 & 10,0 & 42 & 11,5 \\
\hline Medicina & 14 & 13,1 & 25 & 9,7 & 39 & 10,7 \\
\hline Nutrição & 8 & 7,5 & 25 & 9,7 & 33 & 9,0 \\
\hline Psicologia & 7 & 6,5 & 19 & 7,3 & 26 & 7,1 \\
\hline Fonoaudiologia & 4 & 3,7 & 11 & 4,2 & 15 & 4,1 \\
\hline Odontologia & 8 & 7,5 & 5 & 1,9 & 13 & 3,6 \\
\hline Farmácia & 1 & 0,9 & 11 & 4,2 & 12 & 3,3 \\
\hline Outras & 10 & 9,3 & 17 & 6,6 & 27 & 7,1 \\
\hline Não conhecida & 6 & 5,6 & 28 & 10,8 & 34 & 9,3 \\
\hline
\end{tabular}

A tabela 2 mostra a abordagem, desenho, amostra e temática do trabalho. Constam os temas tratados em cinco ou mais manuscritos. Cabe ressaltar que, dentre as revisões, apenas uma realizou uma análise crítica da metodologia dos trabalhos encontrados, sendo classificada, portanto, como revisão sistemática. A média do número de referências foi 28,24 e a moda, 35. A percentagem de referências não atuais, ou seja, anterior a cinco anos da publicação do artigo, foi de $61,54 \%$ durante todo o período. Especificamente, $60,2 \%$ no ano $2006 ; 64,5 \%$ em $2007 ; 62,6 \%$ em 2008; 58,5\% em 2009; 59,2\% em 2010;59,4\% em 2011; 63,8\% em 2012 e 63,3\% em 2013.

Os dez trabalhos da RBGG mais citados estão descritos na tabela 3. Destaca-se que a maior parte são artigos de revisão. 
Tabela 2. Análise descritiva das variáveis referentes aos trabalhos publicados na RBGG entre 2006 e 2013. Natal-RN, 2014.

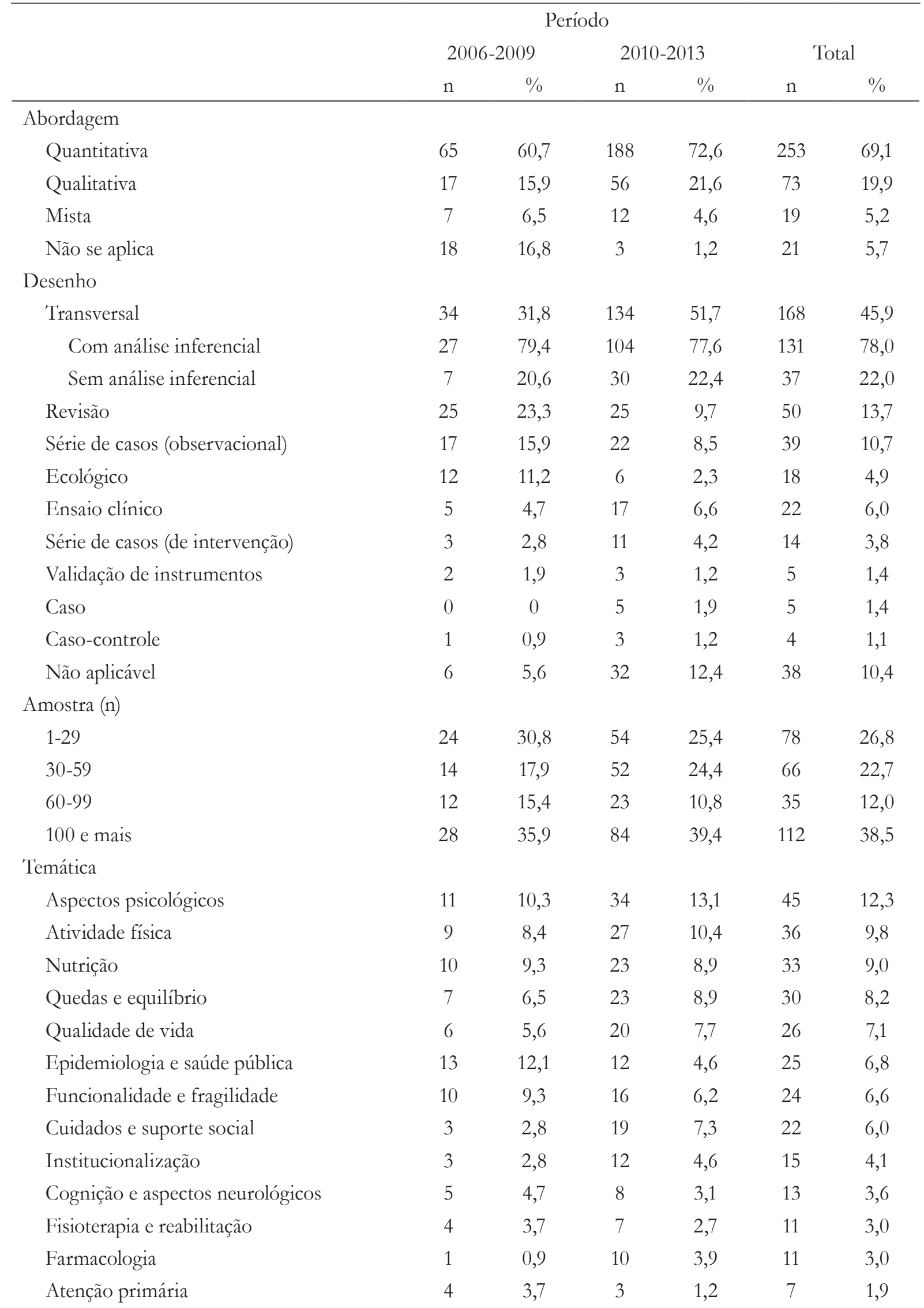




\begin{tabular}{lccccccc}
\hline & \multicolumn{3}{c}{ Período } & \multicolumn{3}{c}{} \\
& \multicolumn{2}{c}{$2006-2009$} & \multicolumn{2}{c}{$2010-2013$} & \multicolumn{2}{c}{ Total } \\
& $\mathrm{n}$ & $\%$ & $\mathrm{n}$ & $\%$ & $\mathrm{n}$ & $\%$ \\
\hline Doenças infecciosas & 2 & 1,9 & 5 & 1,9 & 7 & 1,9 \\
Incontinência urinária & 1 & 0,9 & 6 & 2,3 & 7 & 1,9 \\
Saúde bucal & 2 & 1,9 & 3 & 1,2 & 5 & 1,4 \\
Fisiologia & 4 & 3,7 & 1 & 0,4 & 5 & 1,4 \\
Outras temáticas & 12 & 11,2 & 30 & 11,6 & 41 & 11,2 \\
\hline
\end{tabular}

Tabela 3. Ranking de artigos da RBGG com maior número de citações. Natal-RN, 2014.

\begin{tabular}{|c|c|c|c|}
\hline Citações & Autores & Ano & Título \\
\hline 40 & Almeida, T; Lourenço, ML. & 2007 & $\begin{array}{l}\text { Envelhecimento, amor e sexualidade: utopia ou } \\
\text { realidade. }\end{array}$ \\
\hline 36 & $\begin{array}{l}\text { Jardim, VCFS; Medeiros, BF; } \\
\text { Brito, AM. }\end{array}$ & 2006 & $\begin{array}{l}\text { Um olhar sobre o processo do envelhecimento: a } \\
\text { percepção de idosos sobre a velhice. }\end{array}$ \\
\hline 30 & Pollo, SHL; Assis, M. & 2008 & $\begin{array}{l}\text { Instituições de longa permanência para idosos - } \\
\text { ILPIs: desafios e alternativas no munícipio do Rio } \\
\text { de Janeiro. }\end{array}$ \\
\hline 29 & $\begin{array}{l}\text { Tinoco, ALA; Brito, LF; } \\
\text { Sant'Anna, MSL; Abreu, WC; } \\
\text { Mello, AC; Silva, MMS; et al. }\end{array}$ & 2006 & $\begin{array}{l}\text { Sobrepeso e obesidade medidos pelo índice de } \\
\text { massa corporal (IMC), circunferência da cintura } \\
\text { (CC) e relação cintura/quadril (RCQ), de idosos de } \\
\text { um município da Zona da Mata Mineira. }\end{array}$ \\
\hline 27 & $\begin{array}{l}\text { Vilela, ABA; Meira, EC; Souza, } \\
\text { AS; Souza, DM; Cardoso, IS; } \\
\text { Sena, IS; et al. }\end{array}$ & 2006 & $\begin{array}{l}\text { Perfil do familiar cuidador de idoso doente e/ou } \\
\text { fragilizado do contexto sociocultural de Jequié-BA. }\end{array}$ \\
\hline 24 & $\begin{array}{l}\text { Veras, RP; Caldas, CP; Coelho, } \\
\text { FD; Sanchez, MA. }\end{array}$ & 2007 & $\begin{array}{l}\text { Promovendo a saúde e prevenindo a dependência: } \\
\text { identificando indicadores de fragilidade em idosos } \\
\text { independentes. }\end{array}$ \\
\hline 23 & $\begin{array}{l}\text { Berlezi, EM; Rosa, PV; Souza, } \\
\text { ACA; Scheneider, RH. }\end{array}$ & 2006 & $\begin{array}{l}\text { Comparação antropométrica e do nível de aptidão } \\
\text { física de mulheres acima de } 60 \text { anos praticantes de } \\
\text { atividade física regular e não praticantes. }\end{array}$ \\
\hline 21 & $\begin{array}{l}\text { Santos, ACPO; Silva, CA; } \\
\text { Carvalho, LS; Menezes, MR. }\end{array}$ & 2007 & A construção da violência contra idosos. \\
\hline 21 & $\begin{array}{l}\text { Santos, MRDR; Mendes, } \\
\text { SCSM; Morais, DB; Coimbra, } \\
\text { MPSM; Araújo, MAM; } \\
\text { Carvalho, CMRG. }\end{array}$ & 2007 & $\begin{array}{l}\text { Caracterização nutricional de idosos com } \\
\text { hipertensão arterial em Teresina-PI. }\end{array}$ \\
\hline 21 & $\begin{array}{l}\text { Benedetti, TRB; Binotto, MA; } \\
\text { Petroski, EL; Gonçalves, LHT. }\end{array}$ & 2008 & $\begin{array}{l}\text { Atividade física e prevalência de quedas em idosos } \\
\text { residentes no sul do Brasil. }\end{array}$ \\
\hline
\end{tabular}


A figura 1 mostra a tendência do tempo, em meses, entre manuscrito recebido e aprovado (tempo de aprovação), entre o tempo de aprovado e publicado (tempo de publicação) e o total, durante o período 2006-2013. Já a figura 2 aponta a evolução da quantidade de manuscritos recebidos, recusados e publicados no período 2006-2013. Comparando os anos 2006 e 2013, houve incremento no número de artigos recebidos $(600 \%)$, recusados $(1.666,66 \%)$ e publicados $(245,45 \%)$.

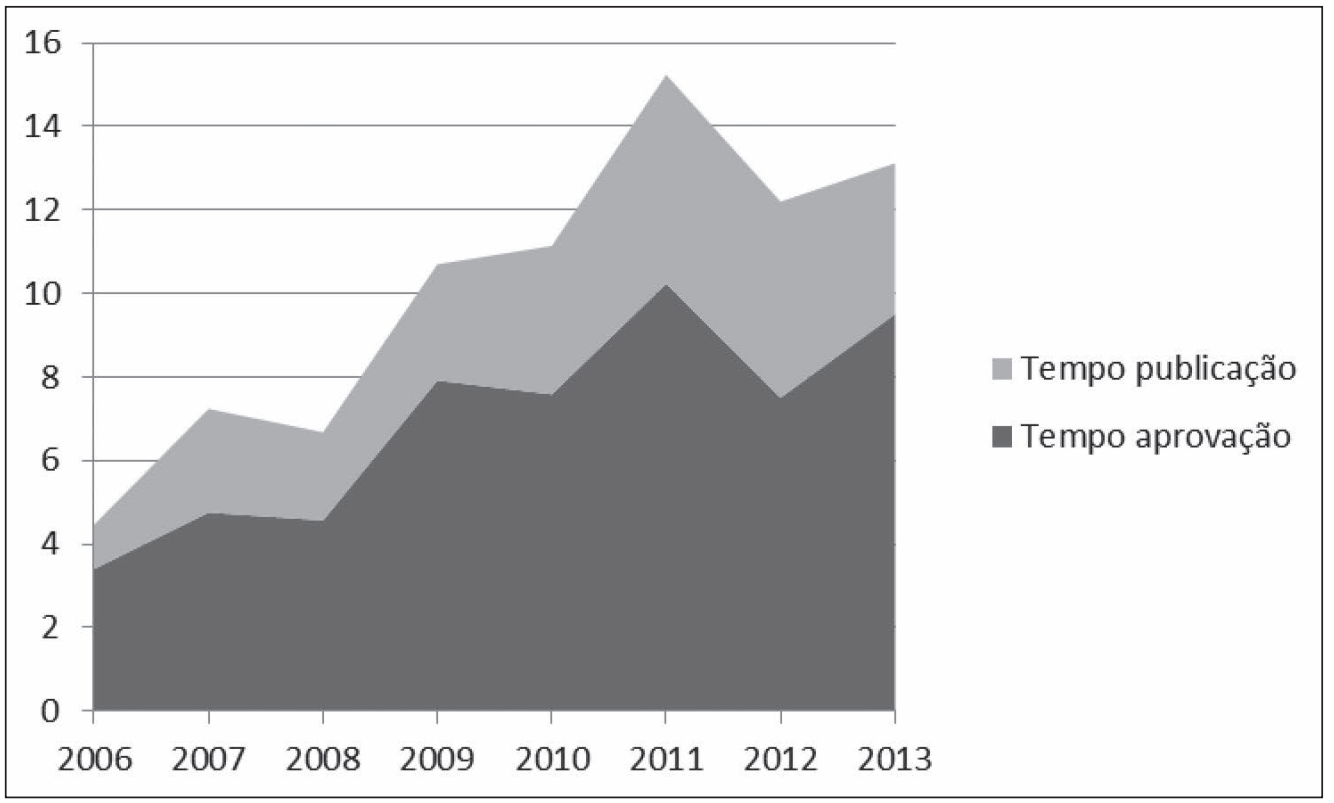

Figura 1. Tendência do tempo de aprovação, publicação e total (em meses) de manuscritos na RBGG, durante o período 2006-2013. Natal-RN, 2014.

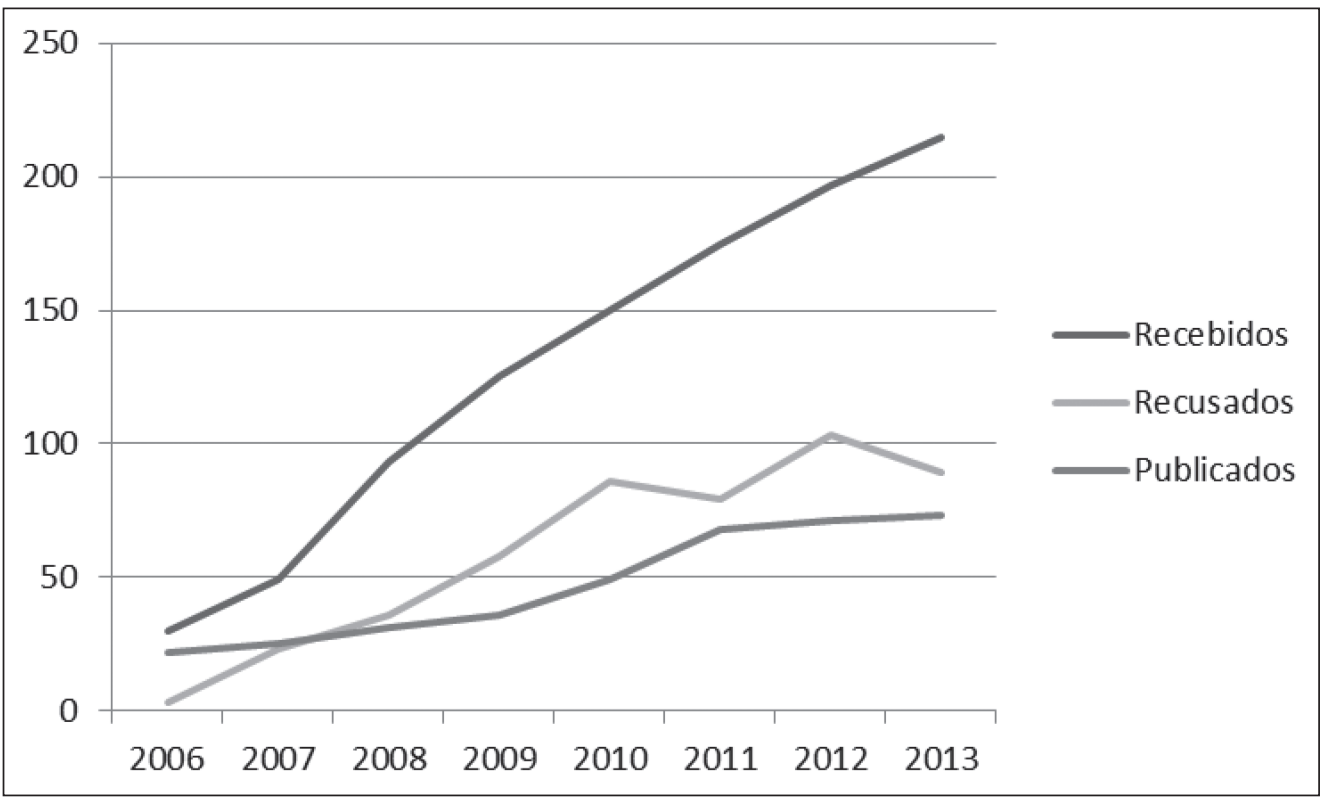

Figura 2. Tendência do número de artigos submetidos, recusados e publicados entre os anos de 2006 e 2013. Natal-RN, 2014. 


\section{DISCUSSÃO}

Os estudos bibliométricos sobre a produção científica em Geriatria e Gerontologia são escassos. Goldstein ${ }^{13}$ realizou um levantamento das teses de doutorado e dissertações de mestrado que enfocam o envelhecimento e a velhice sob o olhar gerontológico, nas diversas áreas do conhecimento, realizadas por pesquisadores brasileiros nos últimos 25 anos, avaliando os temas abordados. Seus achados, apesar de categorizarem as temáticas de forma mais específica, foram ao encontro da maior parte dos resultados do presente estudo. ${ }^{13} \mathrm{O}$ mesmo autor ressalta que o interesse pela temática do envelhecimento passa a abranger diferentes domínios disciplinares, revelando diferentes faces da velhice. Concomitante a isso, verificouse uma expansão na diversidade dos temas abordados, fato que também foi identificado neste trabalho. ${ }^{13}$

Ainda sobre as temáticas em Geriatria e Gerontologia, o presente estudo apresentou na produção científica na RBGG cinco temas em destaque: aspectos psicológicos; atividade física; nutrição; quedas e equilíbrio; e qualidade de vida. Esses componentes vêm contemplar demandas mais atuais e abrangentes sobre o conceito de saúde, levando em conta não somente aspectos biológicos, mas também psicológicos, sociais e de qualidade de vida. ${ }^{14}$

Sobre a produção científica no Brasil, nas diversas temáticas relativas ao envelhecimento e mesmo a outras áreas de estudos, identificouse sua íntima ligação com o ensino superior e programas de pós-graduação, que se desenha em concordância com o desenvolvimento das universidades de forma localizada e centralizada em nosso país. ${ }^{15}$ Franco ${ }^{15}$ ressalta que o ensino superior no Brasil iniciou sua organização mais sistemática a partir de 1934, com a fundação da Universidade de São Paulo. Esse evento representa uma condição histórica recente no cenário educacional, bem como o desenvolvimento de outras instituições de forma centralizada na Região Sudeste. ${ }^{15,16}$
Cabe ressaltar que não se encontrou manuscrito proveniente de instituição do ensino básico e/ou técnico. A baixa produção científica nestes níveis de educação limita a produtividade, sobrecarrega a pesquisa nos níveis mais elevados de educação e não estimula uma cultura inovadora de formação de pesquisadores desde os níveis mais básicos do ensino.

Guimarães $^{12}$ destaca que a distribuição geográfica das atividades de pesquisa em saúde acompanha um padrão de concentração regional observado para o conjunto das atividades de pesquisa no país: 63\% dos grupos estão localizados na Região Sudeste, 17\% no Sul, 13\% no Nordeste, $5 \%$ no Centro-Oeste e $2 \%$ no Norte. ${ }^{12}$ Esses achados corroboram os resultados deste trabalho, sugerindo que as regiões de destaque podem ser consideradas áreas de excelência na produção científica, nas linhas de pesquisa em Geriatria e Gerontologia. ${ }^{12,17,18}$

A concentração de produção científica, também identificada entre as instituições de ensino superior, reflete-se em outras análises de produtividade, como na lista das 500 universidades mais produtivas do mundo elaborada pela Universidade de Comunicações de Xangai (Shangai Jiao Tong University - SJTU). ${ }^{19}$ O mesmo trabalho publicado em 2011 divulga uma lista das melhores universidades do mundo e é elaborada com base em critérios de produção científica desde 2003. Dentre estas, constam seis universidades brasileiras: Universidade de São Paulo - USP (entre as 101 e 150 posições), seguida pela Universidade Estadual de Campinas, Universidade Federal de Minas Gerais (UFMG), Universidade Federal do Rio de Janeiro (UFRJ), Universidade Estadual Paulista - UNESP (entre as 201 e 300 posições) e a Universidade Federal do Rio Grande do Sul - UFRGS (entre as 401 e 500 posições).

Cabe destacar que, dentre as brasileiras citadas, quatro apareceram no ranking de instituições com maior número de publicações na RBGG entre os anos de 2006 e 2013, demonstrando que este ranqueamento obteve adequada reprodutibilidade 
no que se refere à produtividade científica no país, apontando assim as instituições com maior nível de produção científica, no que se refere à publicação de artigos. ${ }^{19}$

Apesar da maior concentração nas regiões Sul e Sudeste, é interessante salientar que a revista tem conseguido transpor as barreiras geográficas na divulgação do conhecimento científico. Exemplo disso, é que o estado do Rio de Janeiro, localização física da revista, não foi o estado com maior número de publicações, havendo inclusive redução considerável destas publicações nos últimos anos. Embora o número de estudos estrangeiros ainda seja reduzido se comparado com as pesquisas nacionais, este se manteve durante todo o período.

Quanto ao número de autores, verificou-se um crescimento nos manuscritos publicados com três ou mais autores, e um decréscimo dos manuscritos com apenas um e dois autores entre os períodos de 2006-2009 e 2010-2013, fator que mostra uma possível integração do conhecimento diversificado com um número maior de pesquisadores. Já no ranking de autores mais citados na literatura, observou-se que os temas estudados por eles foram genéricos e abrangentes, sendo publicados entre os anos de 2006 e 2007, possivelmente por serem artigos disponíveis na internet por um período de tempo maior e por se tratar de temáticas de interesse de estudo atual.

No que diz respeito à metodologia dos trabalhos analisados, destacam-se os estudos quantitativos do tipo transversal, com amostras com 100 sujeitos ou mais e com análise inferencial. Machado ${ }^{20}$ destaca a força da validade externa dos estudos quantitativos, onde seus resultados, normalmente provenientes de procedimentos controlados, podem ser generalizáveis para a comunidade e replicáveis. Santos \& Victora ${ }^{21}$ discutem a importância dos estudos seccionais, sobretudo na área de Saúde Pública. Esses autores enfatizam que diante de sistemas mais complexos para avaliação, a modificação de efeito por parte de várias características externas à intervenção é altamente provável. Somam-se ainda as condições de pesquisas por ensaios clínicos randomizados: muitas vezes pode ocorrer um controle excessivo sobre a amostra, que não reflete intervenções sob condições de rotina ou é limitado por questões éticas. Desta forma, os estudos seccionais devem ser considerados para complementar os achados dos ensaios clínicos randomizados. ${ }^{21}$

Outro ponto que deve ser questionado se refere ao apoio financeiro por iniciativa pública ou privada: em relação aos artigos publicados com apoio financeiro, lamentavelmente, os índices ainda são bastante reduzidos. Mesmo que o Governo Federal considere que os gastos com a educação superior são maiores que os gastos com a educação básica, considerando a proporção per capita, boa parte destes gastos se refere à manutenção das instituições federais de ensino superior, aos programas de financiamento estudantil e "universidade para todos". Somase a isso o fato de que o financiamento público começou a se desenvolver, de forma mais significativa, somente a partir da década de 1990, o que torna o fomento à pesquisa propriamente dita insuficiente. ${ }^{22}$ Isso é mais um obstáculo para desenvolver pesquisas em nosso país, já que muitos projetos de qualidade têm um custo elevado para sua execução e demandam tempo e dedicação por parte dos pesquisadores, que no geral não recebem bolsas compatíveis para se dedicarem exclusivamente aos projetos.

Sobre a tendência de manuscritos recebidos na RBGG, observa-se um crescimento relevante entre os anos 2006 e 2013, de 30 para 180 artigos recebidos comparativamente entre esses anos (incremento de 600\%), provavelmente devido ao maior prestígio da revista, conseguido graças à indexação em novas bases de dados. Como consequência dessa pressão editorial, o tempo médio de publicação aumentou, trazendo prejuízos no acréscimo de produção científica para os autores, para a própria revista e para a comunidade acadêmica. Merece ser lembrado 
que o número de estudos recusados cresceu proporcionalmente com o número de artigos recebidos, traduzindo assim a manutenção do cuidado com os critérios para seleção dos artigos.

Quanto ao número de citações dos trabalhos da revista, cabe destacar a política de livre acesso (open access) dos trabalhos publicados, fato que pode ter contribuído para a maior disseminação do conhecimento produzido na RBGG. Considerando este fato e a indexação em novas bases de dados, é possível que o número de citações aumente nos próximos anos.

Considerando ainda a questão das bases de dados, merece destaque a indexação na Scientific Electronic Library Online (SciELO), que é um modelo para a publicação eletrônica cooperativa de periódicos científicos na internet. Especialmente elaborado para responder às necessidades da comunicação científica nos países em desenvolvimento - particularmente na América Latina e no Caribe. Esta base se propõe a disponibilizar, em texto integral, artigos e periódicos completos para aumento $\mathrm{da}$ visibilidade e acesso (open access) à ciência brasileira. Além disso, atende a uma antiga demanda referente à operação de bases de dados bibliográficos para não apenas controlar e disseminar a literatura científica, mas também permitir a produção de indicadores que subsidiem estudos de bibliometria sobre a produção científica nacional relevante. ${ }^{11}$

\section{REFERÊNCIAS}

1. Bassit AZ, Witter C. Envelhecimento: objeto de estudo e campo de intervenção. In: WITTER GP, organizador. Envelhecimento: referenciais teóricos e pesquisas. $2^{\mathrm{a}}$ ed. Campinas: Alínea; 2010.

2. Caldas CP. Editorial. Rev Bras Geriatr Gerontol 2007;10(1):1-1.

3. Prado SD, Imbassahy M, Sobral B. Apresentação Temático: Saúde e Condições de Vida do Idoso. Textos Envelhecimento 1998 nov; (1): 1.

4. Veras R. Revista Brasileira de Geriatria e Gerontologia [editorial]. Rev Bras Geriatr Gerontol 2006; 9(1):1-1.

\section{CONCLUSÕES}

Constatou-se um incremento do número de trabalhos publicados na Revista Brasileira de Geriatria e Gerontologia no período 20062013, assim como um aumento exponencial da quantidade de manuscritos recebidos. A maioria dos estudos é de abordagem quantitativa e de tipo transversal. A temática mais comum é a referente aos aspectos psicológicos do envelhecimento, atividade física e nutrição.

A produção científica é principalmente realizada nas instituições universitárias, em âmbito nacional e centralizada nas regiões Sudeste e Sul, especificamente nos estados de São Paulo, Rio Grande do Sul e Rio de Janeiro. Durante o período estudado, se observa uma tendência de maior distribuição da frequência das profissões dos autores principais, o qual mostra a grande diversidade da área da Gerontologia.

\section{AGRADECIMENTOS}

À equipe editorial da RBGG que, gentilmente, nos cedeu os dados específicos dos artigos recebidos, recusados e publicados e à equipe da biblioteca da Faculdade de Odontologia da Universidade Federal do Rio Grande do Norte (UFRN), pelo apoio técnico e revisão do presente manuscrito.
5. Prado SD, Abreu CR. Uma contribuição para o crescimento da ciência no campo geriátrico e gerontológico nacional e internacional. Rev Bras Geriatr Gerontol 2011;14(1):5-6.

6. Veras R. A Revista Brasileira de Geriatria e Gerontologia na Scielo: uma trajetória bem sucedida no campo do envelhecimento [editorial]. Rev Bras Geriatr Gerontol 2012;15(2):1-1.

7. Coordenação de Aperfeiçoamento de Pessoal de Nível Superior. Sistema Integrado Capes. Webqualis [Internet]. Brasília, DF: CAPES; 2014 [acesso em 5 Abr 2014]. Disponível em: http://qualis.capes.gov.br/ 
8. Silva RC. Avaliação da informação científica em Bibliometria aplicada às Ciências da Saúde. In: Bibliotecas, informação, usuários: abordagens de transformação para Biblioteconomia e Ciência da Informação: Anais $25^{\circ}$ do Congresso Brasileiro de Biblioteconomia, Documentação e Ciência da Informação; 2013 Jul 07-10; Florianópolis, SC: FEBAB; 2013. p. 1-16.

9. Araújo CA. Bibliometria: evolução histórica e questões atuais. Rev Questão 2006;12(1):11-32.

10. Mugnaini R. Indicadores bibliométricos da produção científica brasileira: uma análise a partir da base Pascal. Ci Inf 2004;33(2):123-31.

11. Ravelli APX, Fernandes GCM, Barbosa SFF, Simão E, De Santos SMA, Meirelles BHS. A produção do conhecimento em enfermagem e envelhecimento: estudo bibliométrico. Texto \& Contexto Enferm 2009;18(3):506-12.

12. Guimarães R. Desafios da pós-graduação em saúde humana no Brasil. Rev Saúde Pública 2011;45(1):1-13.

13. Goldstein LL. A produção científica brasileira na área da gerontologia: (1975-1999). Rev online Bibl Prof Joel Martins 1999;1(1):1-9.

14. Segre M, Ferraz FC. O conceito de saúde. Rev Saúde Pública 1997;31(5): 538-42.

15. Franco AP. Ensino Superior no Brasil: cenário, avanços e contradições. J Polít Educ 2008;2(4):53-63.
16. Cunha LA. Desenvolvimento desigual e combinado no ensino superior: estado e mercado. Educ Soc 2004;25(88 Esp):795-817.

17. Costa MCMDR, Lima SP, Santos LMC, Silva ER, Erdmann AL. Teoria fundamentada nos dados em pesquisa na saúde da mulher: estudo bibliométrico. Rev enferm UFPE on line 2013;7(Esp):4153-60.

18. Luz MP, Nascimento AL, Mendlowicz M, Appolinario JC, Figueira V. Jornal Brasileiro de Psiquiatria: um estudo bibliométrico dos artigos publicados de 1995 a 2004. J Bras Psiquiatr 2007;56(1):29-32.

19. Hilu L, Gisi ML. Produção científica no Brasil: um comparativo entre universidades públicas e privadas. In: Vosgerau DSR, Ens RT, organizadoras. Formação para mudanças no contexto da educação: políticas, representações sociais e práticas: Anais do $10^{\circ}$ Congresso Nacional de Educação, $1^{\circ}$ Seminário Internacional de Representações Sociais, Subjetividade e Educação; 2011 nov 7-10; Curitiba: Champagnat; 2011. p. 5665-72.

20. Machado MF. Diálogo entre metodologias quantitativas e qualitativas no campo da saúde. Psicol.Pt 2011:1-7.

21. Santos IS, Victora CG. Serviços de saúde: epidemiologia, pesquisa e avaliação. Cad Saúde Pública 2004;20(2):337-41.

22. Corbucci PR. Desafios da educação superior e desenvolvimento no Brasil. Texto Discussão 2007;1287:1-33. 